\title{
Design of SMC Motors Using Hybrid Optimization Techniques and 3D FEA with Increasing Accuracy
}

\author{
YouGuang GUO, Jian Guo ZHU, and Haiwei LU \\ Faculty of Engineering, University of Technology, Sydney, PO Box 123, Broadway, NSW 2007, Australia
}

\begin{abstract}
This paper presents the design and analysis of a three-phase three-stack permanent magnet claw pole motor with soft magnetic composite (SMC) stator core. 3D finite element analysis (FEA) of magnetic field is performed to accurately calculate key motor parameters and performance. Combined optimization techniques and 3D FEA with increasing accuracy are applied to effectively reduce the computational time. The designed motor has been fabricated and tested. The theoretical calculations are validated by the experimental results on the prototype.
\end{abstract}

\section{INTRODUCTION}

The study about soft magnetic composite (SMC) materials and their application in electromagnetic devices has attracted strong interests of research in the past decade, and some encouraging progresses have been achieved. The unique properties of SMC include isotropic magnetic and thermal properties, very low eddy current loss, relatively low total core loss in the medium and higher frequency range, nearly net-shape fabrication process with good tolerance and surface finish, and the prospect of very low cost mass production [1]. Particularly, the magnetically isotropic property of SMC opens up crucial design advantages since the magnetic field does not have to be constrained in a two-dimensional (2D) plane as in conventional laminated machines. Therefore, SMC is very suitable for electromagnetic devices with three-dimensional (3D) fluxes such as claw pole and transverse flux motors [2].

In 3D flux machines, the magnetic flux can spread in any direction and inherently a considerable portion of the total flux is the leakage flux. This is intensified by the relatively low magnetic permeability of SMC. It is essential to use 3D finite element analysis (FEA) for an accurate electromagnetic design.

This paper presents the design and analysis of a threephase three-stack permanent magnet (PM) claw pole motor with SMC stator core. The preliminary design was conducted by the equivalent magnetic circuit with linear approximation. 3D numerical electromagnetic field analysis by finite element method (FEM) was performed to accurately compute the magnetic field distribution and key motor parameters such as the PM flux (defined as the stator winding flux produced by the rotor PMs), back electromotive force (emf), cogging torque and electromagnetic torque, inductance and core losses. Equivalent electric circuit was employed to predict the motor performance. Optimization techniques were used to optimize key dimensions, such as the axial length of magnets, to achieve the minimum ratio of the overall material cost and the electromagnetic torque. Thermal analysis was carried out to check the temperature rise, a key constraint on the motor. Finally, the motor prototype was fabricated and tested.

For the design optimization of the SMC motor, combined techniques are employed to reduce the computational time while keeping a reasonable accuracy. For example, the genetic algorithm (GA) is applied for the global search and then the direct search method and the fine grid-search are carried out around the optimum obtained from the first method.

In the optimization searching for the best design, a large number of 3D FEA iterations are required for electromagnetic design and thermal verification, which could make the computing time too long to be practical. In order to shorten effectively the computing time, a strategy of 3D FEA with increasing accuracy is used. The simple idea is that in the early stages of optimization, simplified formulations for parameter prediction and rough 3D meshes are used, which may be inaccurate but certainly fast. Then the mesh is refined with the progress of optimization. When the optimum is reached the mesh and the parameter formulations should be fine enough for accurate computation of motor parameters and performance.

\section{SMC Claw Pole Motor Prototype}

Electrical machines with claw pole rotors or stators have been manufactured in mass production for many years. These machines have quite simple excitation coil and pole systems producing the excitation magnetic fields. They are capable of producing power densities up to three times greater than conventional machines because the topology allows the pole number to be increased without reducing the magnetomotive force per pole. The excessive eddy currents in the commonly used solid steel core, however, limit the motors to very small sizes and/or low speeds and result in low efficiency.

Because of the complex structure, it is very difficult to construct the claw poles using electrical steel laminations. SMC materials offer an opportunity to overcome these problems. Fig. 1 illustrates the magnetically relevant parts of the rotor and the stator of a claw pole SMC motor prototype [3, 4]. Table 1 lists the dimensions and major parameters. The three phases of the motor are stacked axially with an angular shift of $120^{\circ}$ electrical from each other. Each stator phase has a single coil around an SMC core, which is molded in two halves. The outer rotor comprises a tube of mild steel with an array of magnets for each phase mounted on the inner surface. Mild steel is used for the rotor because the flux density in the yoke is almost constant. 


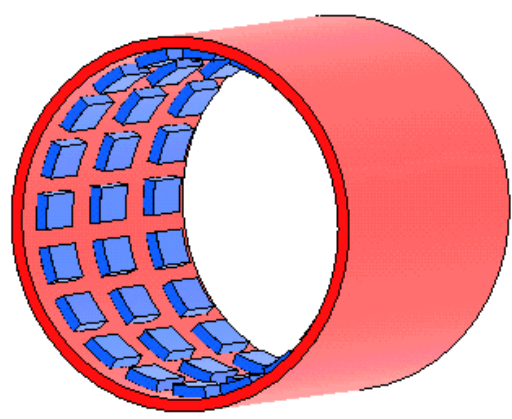

(a)

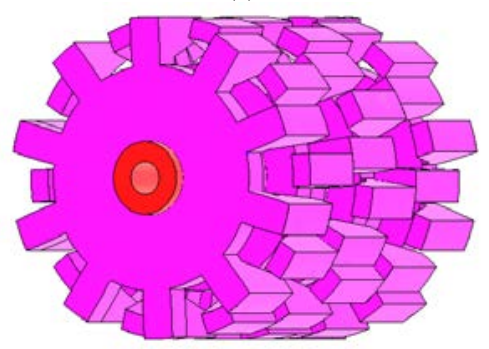

(b)

Fig. 1. The magnetically relevant parts of the claw pole motor: (a) outer rotor, (b) SMC stator.

TABLE I

DimENSIONS AND MAJOR PARAMETERS

\begin{tabular}{ll}
\hline Dimensions and parameters & Quantities \\
\hline Rated frequency $(\mathrm{Hz})$ & 300 \\
Number of phases & 3 \\
Rated power $(\mathrm{W})$ & 500 \\
Rated line-to-neutral voltage $(\mathrm{V})$ & 64 \\
Rated phase current $(\mathrm{A})$ & 4.1 \\
Rated speed (rev/min) & 1800 \\
Rated torque (Nm) & 2.65 \\
Rated efficiency $(\%)$ & 81.5 \\
Rated temperature rise in stator winding $\left({ }^{\circ} \mathrm{C}\right)$ & 75 \\
Number of poles & 20 \\
Stator core material & $\mathrm{SMC}$ \\
Stator outer radius (mm) & 40 \\
Effective stator axial length $(\mathrm{mm})$ & 93 \\
Total motor length (mm) & 137 \\
Rotor outer radius (mm) & 47 \\
Rotor inner radius $(\mathrm{mm})$ & 41 \\
Permanent magnets & $\mathrm{NdFeB}$ \\
Number of magnets & 60 \\
Magnet dimensions & OD88 x ID82 x 15 \\
& mm arc $12^{\circ}$ \\
Magnetization direction & Radial \\
Main airgap length $(\mathrm{mm})$ & 1 \\
Stator shaft material & Mild steel \\
Shaft outer radius (mm) & 9 \\
Number of coils & 3 \\
Coil window dimension $\left(\mathrm{mm}^{2}\right)$ & $17 \times 11$ \\
Number of turns & 75 \\
Number of strands & 2 \\
Diameter of copper wire $(\mathrm{mm})$ & 0.71 \\
Resistance per phase at $115^{\circ} \mathrm{C}(\Omega)$ & 0.302 \\
\hline
\end{tabular}

\section{3D FEA OF MAGNETIC FIELD}

It is evident that the complicated shape of a claw pole machine leads to a truly 3D magnetic flux. Therefore, it is necessary that 3D finite element analysis be conducted for accurate determination of the parameters and performance of the electrical machine.

\section{A. FEA Solution Region}

The magnetic circuits of three stacks (or phases) of the motor are basically independent. For each stack, because of the symmetrical structure, it is only required to analyze the magnetic field in one pole pitch, as shown in Fig. 2.

At the two radial boundary planes of one pole pitch, the magnetic scalar potential obeys the so-called halfperiodical boundary conditions:

$$
\varphi_{m}(r, \Delta \theta / 2, z)=-\varphi_{m}(r,-\Delta \theta / 2,-z)
$$

where $\Delta \theta=18^{\circ}$ is the angle of one pole pitch. The original point of the cylindrical coordinate is located at the center of the stack.

\section{B. FEA of Magnetic Field}

Fig. 3(a) illustrates the no-load flux density vectors with line lengths proportional to the magnitudes at rotor position $\theta=0^{\circ}$, defined as where the magnets share the same axes as the stator claw poles respectively. It is shown that the major path of the magnetic flux of the PMs is along one of the PMs - the main air gap - one of the SMC claw pole stator core pieces - the SMC stator yoke - another SMC claw pole stator core piece - main airgap - another PM and then - the mild steel rotor yoke to form a closed loop. There is also a significant amount of leakage flux, especially between the side surfaces of claw poles. Fig. 3(b) plots the flux density vectors generated by the stator current only, showing that the major flux path of the armature reaction is different from that of the magnetic field produced by the PMs.

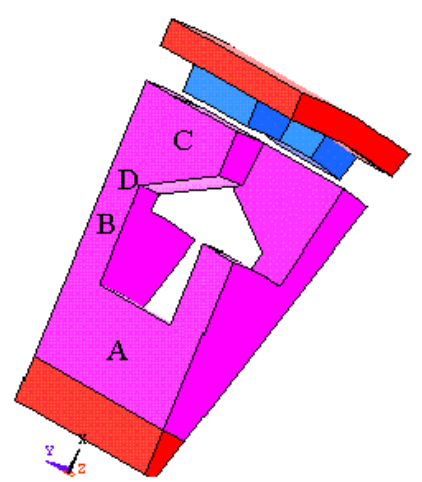

Fig. 2. Region for FEA solution of magnetic field.

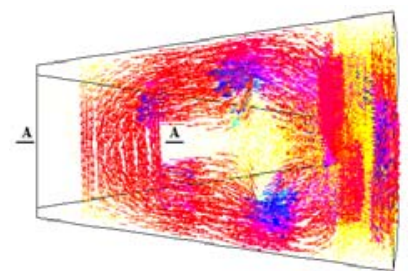

(a)

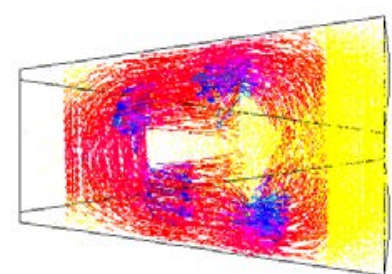

(b)
Fig. 3. Flux density vectors by (a) PM only, (b) stator current only. 


\section{Stator Winding Flux and Back emf}

After the magnetic field distribution is solved, the stator coil flux can be calculated by the surface integration of flux density on a specified cross-sectional area, e.g. A-A in Fig. 3(a). Fig. 4 shows the no-load flux waveform, calculated by rotating the rotor for one pole pitch in 12 steps. This flux waveform versus the rotor position is almost perfectly sinusoidal. When the rotor rotates, a back emf is induced in the winding and can be calculated by differentiating the flux linkage with respect to time.

The PM flux, produced by the rotor PMs, may be deviated at load due to the stator current if the magnetic circuit is saturated. To consider the effect of the armature reaction, two steps of analyses are performed. Firstly, non-linear analysis is conducted with both the stator current and PMs to determine and save the permeability in each element. Next, a linear analysis with the saved permeabilities and PMs only to find the PM flux.

\section{Winding Inductance}

The winding inductance is one of the key parameters determining the motor performance. It can be computed by the flux-linkage method as

$$
L=\frac{N \phi}{i}
$$

or the energy method as

$$
L=\frac{2 W_{f}}{i^{2}}
$$

where $\phi$ and $W_{f}$ are the magnitude of the flux linking the winding and the magnetic co-energy stored in the whole machine, respectively, produced by a current $i$ in each of $N$ turns. The flux and magnetic co-energy can be obtained from the results of a field analysis with a current $i$ while the PMs are "switched off", i.e. remanence is set to zero. The mutual inductance between phases can be considered as zero because of the almost independent magnetic flux paths.

However, this calculation is not accurate if the magnetic circuit is saturated because the saturation is determined by both the stator current and the rotor PMs. To consider the effect of PMs on the saturation, like the PM flux calculation, two steps of analyses are performed, i.e. a non-linear analysis with both the stator current and PMs to determine the operating point and save the permeability in each element, and then a linear analysis with the saved permeabilities and a stator current $i$ only to find the $\phi$ in (2) or the $W_{f}$ in (3). The inductance is then calculated by (2) or (3).

The above calculation is in fact the secant inductance, e.g. along the cord line through the operating point and the origin in the B-H curve. However, the behavior of the electrical circuit is determined by the so-called incremental (or differential) inductance, e.g. along the tangential line of the operating point $[5,6]$. The incremental inductance can also be computed by the above two-step analyses except that the differential permeabilities, rather than the apparent ones, are estimated and saved in step 1, and then applied in step 2. Fig. 5 shows the computed secant and incremental inductances of the phase winding against rotor position when the motor is operated under the optimum brushless DC control condition, i.e. the stator current is controlled in phase with the back emf, at noload, half-load and full-load, respectively.

\section{E. Core Losses}

Due to the complex shape of the claw pole machine, the flux density locus at one position can be very complex, e.g. one-dimensional alternating, 2D or even 3D rotating with purely circular or elliptical patterns, all with or without harmonics. These patterns are affected by the armature reaction. Fig. 6 illustrates the flux density locus in a typical element in the middle of the claw pole at different loads. It is seen that all the patterns are truly 3D and rotating elliptically.

It is known that different flux density patterns have very different effects on the core losses. A comprehensive method [7, 8] is applied to predict the core losses in the 3D flux SMC machine. Different formulations are used for core loss prediction with purely alternating, purely circular rotating, and elliptically rotating flux density vectors, respectively. A series of 3D FEAs are conducted to determine the flux density locus in each element when the rotor rotates (an example is shown in Fig. 6). At the rated speed, the total core loss is calculated as $58.0 \mathrm{~W}$ at noload, but goes up to $67.4 \mathrm{~W}$ at half-load, and further to $81.8 \mathrm{~W}$ at full-load. The core loss increment is nonlinearly related to the armature reaction, e.g. 4.6 W/A from no-load to half-load, and 7.0 W/A from half-load to full-load.

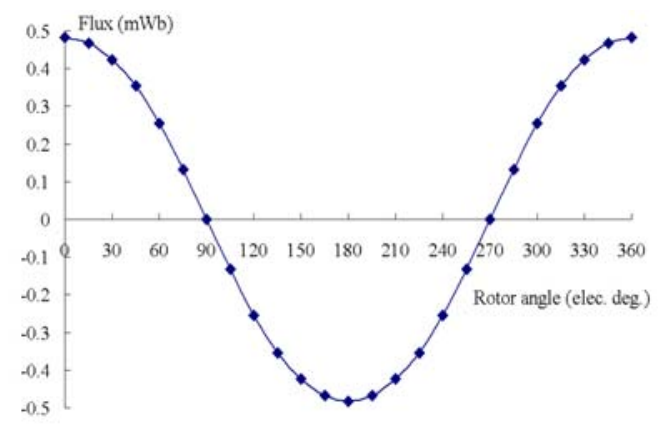

Fig. 4. Calculated on-load winding flux versus rotor angle.

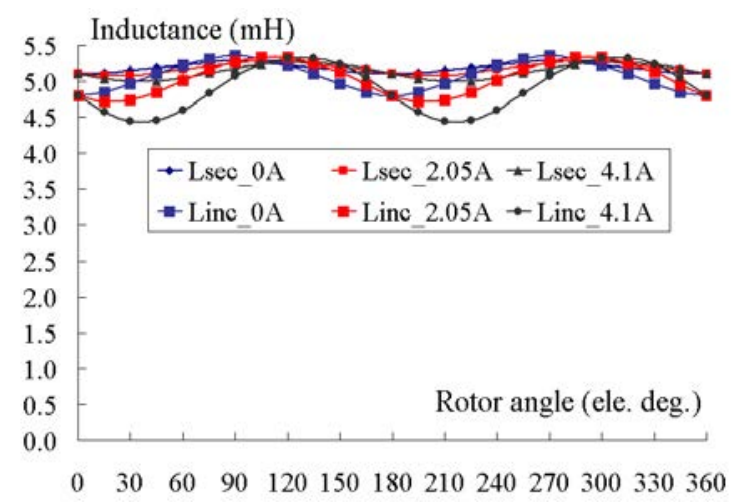

Fig. 5. Computed secant and incremental inductances while FEA considers the saturation caused by both stator current and PMs. 


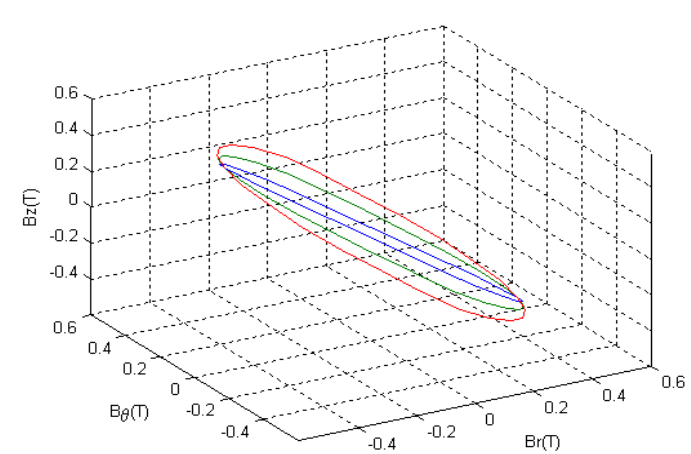

Fig. 6. Flux density loci at Point C of Fig. 2: inner locus for no-load, middle one for half-load, and outer one for full-load.

\section{E. Performance Prediction}

With a closed-loop control of rotor position feedback the motor can operate in brushless DC mode where the current $I_{1}$ (in rms value) and the induced emf, $E_{1}$ (in rms value) in the stator winding are in phase, to achieve a maximum electromagnetic power $P_{e m}$ at a given speed, as

$P_{e m}=3 E_{1} I_{1}$

The induced stator emf can be determined by

$$
E_{1}=\frac{\omega_{1} N_{1} \phi_{m}}{\sqrt{2}}
$$

where $\omega_{1}=2 \pi f_{1}$ is the angular rotor speed in electrical radians per second, $f_{1}$ the frequency of the induced stator emf in Hertz, $N_{1}$ the number of turns of the stator winding, and $\phi_{m}$ the magnitude of the fundamental of the PM flux curve against rotor position.

The rated line-to-neutral rms voltage is

$$
V_{1}=\sqrt{\left(E_{1}+I_{1} R_{1}\right)^{2}+\left(I_{1} X_{1}\right)^{2}}
$$

where $R_{1}$ is the resistance of one phase winding, $X_{1}=\omega_{1} L_{1}$ is the reactance, and $L_{1}$ is the phase winding inductance.

The output power, output torque, input power, and efficiency are calculated by

$$
\begin{aligned}
P_{\text {out }} & =P_{\text {em }}-P_{\mathrm{Fe}}-P_{\text {mec }} \\
T_{\text {out }} & =P_{\text {out }} / \omega_{r} \\
P_{\text {in }} & =P_{\text {em }}+P_{\text {cu }} \\
P_{\text {cu }} & =3 I_{1}^{2} R_{1} \\
\eta & =P_{\text {out }} / P_{\text {in }}
\end{aligned}
$$

where $P_{F e}$ is the core loss, $P_{\text {mec }}$ the mechanical loss, $P_{c u}$ the copper loss, and $\omega_{r}$ the angular speed.

\section{E. Thermal Calculation}

To obtain an economic utilization of the materials and safe operation of the motor it is necessary to predict with reasonable accuracy the temperature rise of the internal parts, especially in the coils and magnets. In this paper, the temperature rise is calculated by using a hybrid thermal network with distributed heat sources [9], as schematically illustrated in Fig. 7.

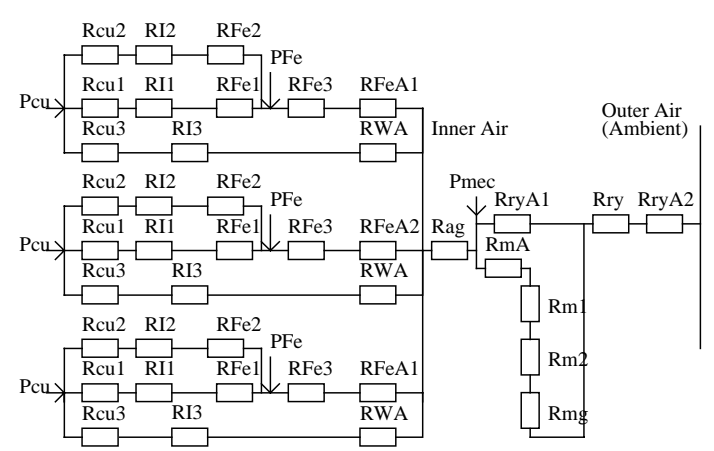

Fig. 7. A schematic diagram of the hybrid thermal network with distributed heat sources [9].

The hybrid model has the advantage of flexibility with reasonable accuracy and computational time, comparing with the conventional thermal network method and the finite element method.

\section{Performance Optimization}

An optimization routine for the design of the claw pole motor with SMC stator core has been set up, which includes three magnetic field FEAs for calculation of PM flux (back emf), stator winding inductance, and core loss, respectively, as described in Section III. The motor performance is predicted based on the optimum brushless DC control condition. The magnetic field FEAs, performance prediction, thermal analysis (by the hybrid model or FEA), and optimization searches are all implemented in a commercial comprehensive software package, ANSYS.

The dimensional variables are the motor outer diameter, effective axial length, rotor yoke thickness, PM dimensions (length, width and height), stator claw pole's axial length, width and height at top and at bottom respectively, and stator yoke thickness. Considering the mechanical clearance requirement, the airgap length is chosen as $1 \mathrm{~mm}$ although smaller value could provide better electromagnetic performance. The design objective is to minimize the total drive cost including main materials (PMs, iron, copper) and power electronics within the temperature rise limit.

In the search for the optimum design, a large number of FEAs are needed and the computational time could be too long to be practical. To effectively reduce the computational time, two techniques can be used, i.e. the hybrid optimization method and FEA with increasing accuracy.

\section{A. Hybrid Optimization Technique}

The most initial and direct method is the grid search, which searches each design variable with its limitation in a very fine step. Although it is the most reliable method, the grid search has been rarely used in practical problems due to the overwhelming computation time, except that only very few (one or two) variables are considered. For example, to check the effect of the magnet axial length on the motor's specific cost, i.e. the ratio of major material cost and electromagnetic torque, a series of analyses (including magnetic field analyses, performance prediction, 
validation of thermal and other constraints, etc.) are conducted for each magnet length, while other dimensions are fixed. This technique is sometimes used to assist the traditional designs, which are done by empirical formulae, previous experience and designers' expertise.

Besides the grid search, two groups of optimisation technique have been developed by various researchers: (1) Classical methods such as the direct search, the simplex method, and the Rosenbrock algorithm; (2) Stochastic methods such as the genetic algorithm and the simulated annealing technique. Compared with the techniques in the second group, those in the first group are generally faster but less safe in determining the global optimum of the objective function [10].

To take the advantages of various optimization techniques while avoiding their disadvantages, the hybrid method can be used. For example, stochastic methods are applied for the global search to find possible optimum region, and then the direct search method and/or the fine grid-search are conducted around the region.

\section{B. FEA with Increasing Accuracy}

The idea of FEA with increasing accuracy is that in the early design stage, rough meshing and simplified parameter formulations are used. This will significantly reduce the computational time although the calculation may not be accurate. The meshing density increases with the design process. When the design reaches the optimum, the meshing and parameter formulations should be fine enough so that the computations of motor parameters and performance are correct.

The above formulations and procedures of parameter calculation can be simplified for fast computation at the first stage. For example, the magnitude of the fundamental of the winding flux produced by the rotor PMs can be approximated by using the flux calculated at no-load at $\theta$ $=0^{\circ}$, because the FEAs have revealed that the curve of the PM flux against rotor angle is almost sinusoidal (Fig. 4), and the effect of armature reaction on the curve is not significant, i.e. only about $3 \%$ reduction in the magnitude of the fundamental of the PM flux due to the rated stator current [11].

\section{EXPERIMENTAL VALIDATION}

The designed SMC motor has been fabricated and operated successfully with a sensorless BLDC controller, delivering a torque of $2.65 \mathrm{Nm}$ at $1800 \mathrm{rev} / \mathrm{min}$. The parameter computations using the magnetic field FEAs are validated by the experimental results on the prototype. For example, Fig. 8 shows the calculated and measured inductances, where the measurement was conducted by the AC voltage-current method with the rotor locked [11]. Fig. 9 compares the calculation and measurement of noload core losses of the motor [4]. The computations agree with the experimental results.

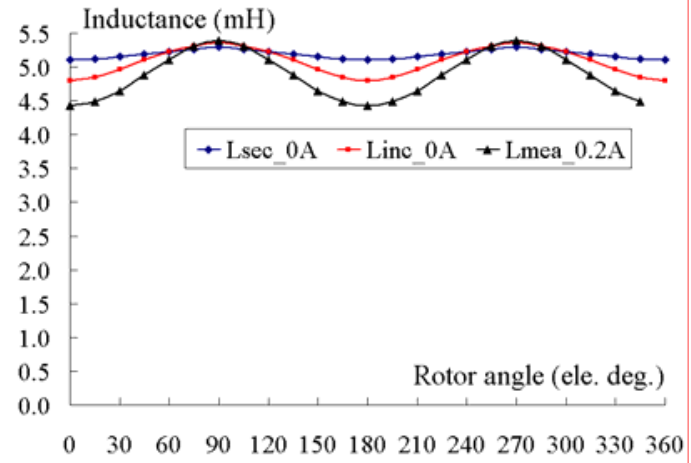

Fig. 8. Computed and measured inductances.

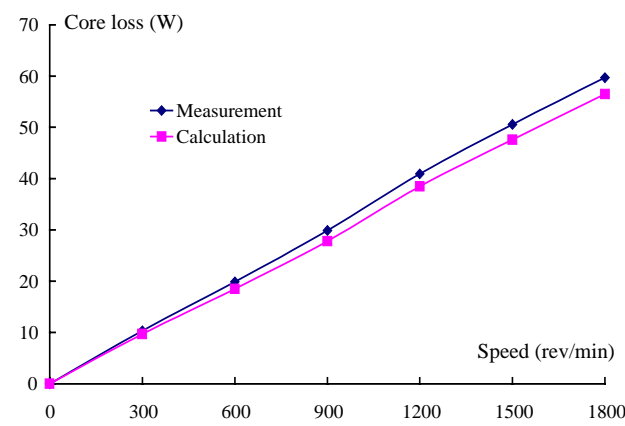

Fig. 9. Computed and measured no-load core losses of a three phase PM claw pole SMC motor.

\section{CONCLUSION}

To investigate the application potential of SMC materials in electrical machine, a three-phase three-stack permanent magnet claw pole motor with SMC stator core has been designed, fabricated and tested. The design makes use of the advantages of 3D magnetic field FEA for accurate computation of the motor parameters and performance. To effectively reduce the computational time, combined optimization techniques and FEA with increasing accuracy are applied in the design optimization. The parameter computations by FEAs agree with the measurements on the prototype.

\section{REFERENCES}

[1] “The latest development in soft magnetic composite technology," SMC Update, Reports of Höganäs AB, Sweden, 1997-2005. Available at http://www.hoganas.com/, see News then SMC Update.

[2] Y.G. Guo, J.G. Zhu, P.A. Watterson, and W. Wu, "Comparative study of 3-D flux electrical machines with soft magnetic composite core,” IEEE Trans. Ind. Applicat., vol. 39, no. 6, pp. 1696-1703, Nov./Dec. 2003.

[3] Y.G. Guo, J.G. Zhu, P.A. Watterson, W.M. Holliday, and W. Wu, "Improved design and performance analysis of a claw pole permanent magnet SMC motor with sensorless brushless DC drive," in Proc. $5^{\text {th }}$ IEEE Int. Conf. on Power Electronics and Drive Systems, Singapore, 17-20 Nov. 2003, pp. 704-709.

[4] Y.G. Guo, J.G. Zhu, P.A. Watterson, and W. Wu, "Development of a claw pole permanent magnet motor with soft magnetic composite stator,” Australian J. of Electrical \& Electronic Eng., vol. 2, no.1, pp. 21-30, 2005.

[5] N.A. Demerdash and T.W. Nehl, "Electrical machinery parameters and torques by current and energy perturbations from field computations - part I: theory and formulation,” IEEE Trans. Energy Conversion, vol. 14, pp. 1507-1513, 1999. 
[6] M. Gyimesi and D. Ostergaard, "Inductance computation by incremental finite element analysis,” IEEE Trans. Magn., vol. 35, no. 3, pp. 1119-1122, May 1999.

[7] Y.G. Guo, J.G. Zhu, J.J. Zhong, and W. Wu, "Core losses in claw pole permanent magnet machines with soft magnetic composite core,” IEEE Trans. Magn., vol. 39, no. 5, pp. 3199-3201, Sept. 2003.

[8] Y.G. Guo, J.G. Zhu, J.J. Zhong, P.A. Watterson, and W. Wu, “An improved method for predicting magnetic power losses in SMC electrical machines,” Int. J. Applied Electromagnetics and Mechanics, vol. 19, pp. 75-78, 2004.
[9] Y.G. Guo, J.G. Zhu, and W. Wu, "Thermal analysis of SMC motors using a hybrid model with distributed heat sources," IEEE Trans. Magn., vol. 41, no. 6, pp. 2124-2128, June 2005.

[10] N. Bianchi and S. Bolognani, "Design optimisation of electrical motors by an adaptive model based optimisation technique," in Proc. IEE Int. Conf. on Electrical Machines and Drives, 1999, pp. 291-296.

[11] Y.G. Guo and J.G. Zhu, "Effect of armature reaction on the performance of a claw pole motor with soft magnetic composite stator by finite element analysis," in Proc. $15^{\text {th }}$ Conf. on the Computation on Electromagnetic Fields, 26-30 June 2005, Shenyang, China. 